\title{
Pulmonary function tests leading to the diagnosis of vascular malformations in school-aged children
}

The authors declare no financial disclosure

\begin{abstract}
Vascular rings are congenital defects of great vessels. They cause compression of the trachea or/and the esophagus and therefore result in symptoms like inspiratory stridor, cough, wheezing, recurrent respiratory tract infections or dysphagia. In some cases, the correct diagnosis can be delayed for many years, especially when vascular rings produce less severe symptoms or symptoms mimicking other diseases (most of all asthma). In this article, we would like to emphasis the usefulness of pulmonary function test (spirometry) in the initial diagnosis of vascular rings in school-aged children, as well as the importance of spirometry in evaluating patients with asthma presumption.
\end{abstract}

Key words: vascular rings, pulmonary function test, spirometry

Adv. Respir. Med. 2017; 85: 253-257

\section{Introduction}

Vascular rings are congenital defects of great vessels. They develop due to abnormal formation of the aortic arch complex. They can be either anatomically complete rings (i.e. double aortic arch) or anatomically incomplete or partial rings, and then are called slings (i.e. pulmonary sling). They cause compression of the trachea or/and the esophagus and therefore result in symptoms like inspiratory stridor, cough, wheezing, recurrent respiratory tract infections or dysphagia [1-4]. Diagnosis is usually established in the first year of life [2] — but in some cases, the correct diagnosis can be delayed for many years, especially when vascular rings produce less severe symptoms or symptoms mimicking other diseases (most of all asthma). In this article, we would like to emphasis the usefulness of pulmonary function test (spirometry) in the initial diagnosis of vascular rings in school-aged children.

\section{Case reports}

\section{Case 1}

A seven-year-old boy was admitted to our hospital because of chronic wet cough and recurrent respiratory tract infections. His health problems had started in early infancy. In the first year of life he was hospitalized multiple times due to bronchiolitis/bronchitis with dyspnea and wheezing, so initial diagnosis of asthma was established and treatment with inhaled corticosteroids was administered. The effect of the therapy was mediocre. Between the age of 1 and 5, the boy still had recurrent respiratory tract infections but they were less frequent and could have been treated at home. The parents reported that doctors were not always convinced what type of respiratory disease their child had. The most common diagnoses were just viral wheezing, recurrent cough or bronchitis. Each infection needed multiple visits at doctor's office and changes in treatment.

Address for correspondence: Beata Kusak, Department of Pediatrics, Chair of Pediatrics, Jagiellonian Univeristy Medical College, Krakow, Poland, Wielicka 265, 30-663 Kraków, tel. 12658 2011, 1655; fax. +48 12658 2426; e-mail: beatakusak@gmail.com DOI: 10.5603/ARM.2017.0042

Received: xx.xx.2017

Copyright (C) 2017 PTChP

ISSN 2451-4934 


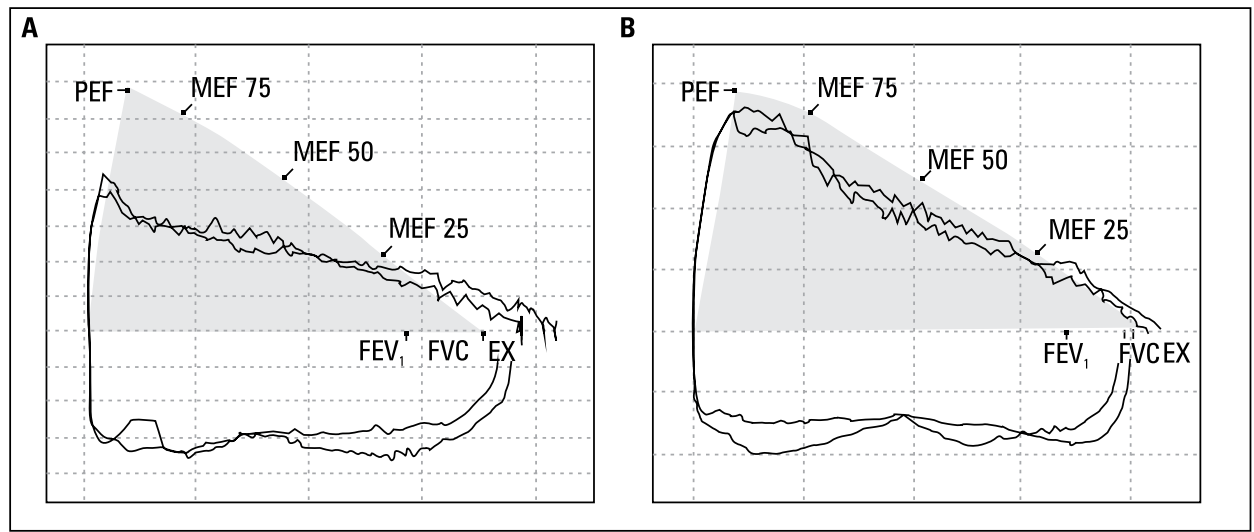

Figure 1. A - the flow-volume curve (before and $15 \mathrm{~min}$ after inhalation of salbutamol) with a plateau of forced expiratory and the inspiratory limb suggesting a fixed central obstruction; B — the spirometry 10 months after the surgery

Table 1. Case 1 - values of spirometry parameters

\begin{tabular}{lcccc}
\hline & \multicolumn{2}{c}{ Before surgery } & 10 months after surgery \\
\cline { 2 - 5 } & \% of predicted value & $\begin{array}{c}\text { Standardised } \\
\text { Z-scores }\end{array}$ & \% of predicted value & $\begin{array}{c}\text { Standardised } \\
\text { Z-scores }\end{array}$ \\
\hline FEV ${ }_{1}$ & 89 & -1.38 & 104 & -1.38 \\
$\mathrm{FVC}$ & 110 & +1.45 & 103 & +0.49 \\
$\mathrm{FEV}{ }_{1} \% \mathrm{FVC}$ & 72 & -3.66 & 92 & -1.06 \\
\hline
\end{tabular}

The child was usually treated with antibiotics and systemic corticosteroids. The most constant sign of those infections was prolongated cough (about 4-5 weeks).

At the age of 6 , the boy started attending school and triggered his infections again. In one year, he underwent 2 episodes of pneumonia, 2 episodes of bronchitis and 3 episodes of laryngitis, besides that, once a month he had upper respiratory tract infection. The main sign of all these infections was barking, hollow, weird cough. At some point, his cough became wet with bringing up sputum (not always productive), especially in the morning. The parents also reported cough during and after exercising.

On the admission day to our hospital, the boy was in a good condition, with normal height and slightly overweight, without dyspnea. We started our diagnostic approach with spirometry (Fig. 1A, Table 1), which showed abnormalities in the flow-volume curve. A plateau of forced expiratory flow and the inspiratory limb was presented suggesting a fixed central airway obstruction. A flexible bronchoscopy was performed and significant malacia affected the distal end of the trachea, the proximal end of the main right bronchus and the whole left main bronchus was revealed (Fig. 2A). Chest computed tomography (CT) with intravenous contrast finally identified the cause - there was abnormal course of the left pulmonary artery in the mediastinum. The left pulmonary artery arose from the pulmonary trunk, then passed the esophagus on his right side and went between the esophagus and trachea and then alongside the main left bronchus forming pulmonary sling (Fig. 2B).

The child underwent surgery - the left pulmonary artery re-implantation was carried out via median sternotomy. Three months after surgery the child was free of weird-sounding cough, he still had cough on exertion and morning wet cough (always effective). Ten months after surgery he presented no symptoms. Furthermore, his spirometry gradually improved (Fig. 1B, Table 1).

\section{Case 2}

An eleven-year-old girl was admitted to our hospital because of chronic cough and recurrent respiratory tract infections. Her health problems had started around the age of 5 . She suffered from chronic rhinitis and recurrent bronchitis (app. 1 episode every 2 months), they were accompanied by wheezing in the last two years. The infections were of mild severity and the girl was never ad- 

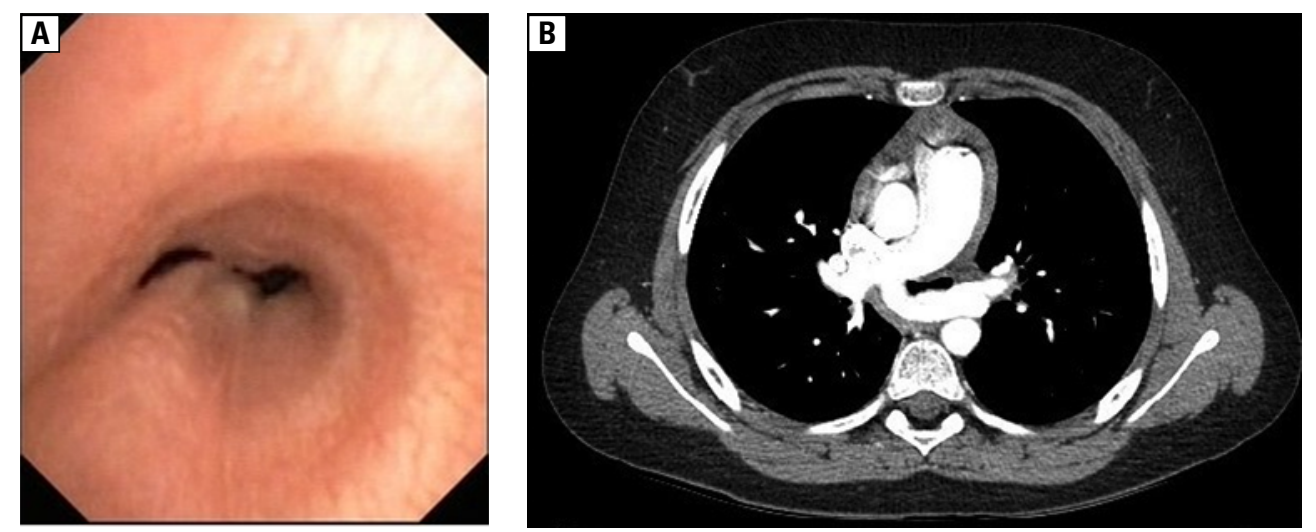

Figure 2. A — flexible bronchoscopy — significant malacia affected distal end of the trachea, proximal end of the main right bronchus and the whole left main bronchus; B - CT scan — abnormal course of the left pulmonary artery in the mediastinum forming pulmonary sling

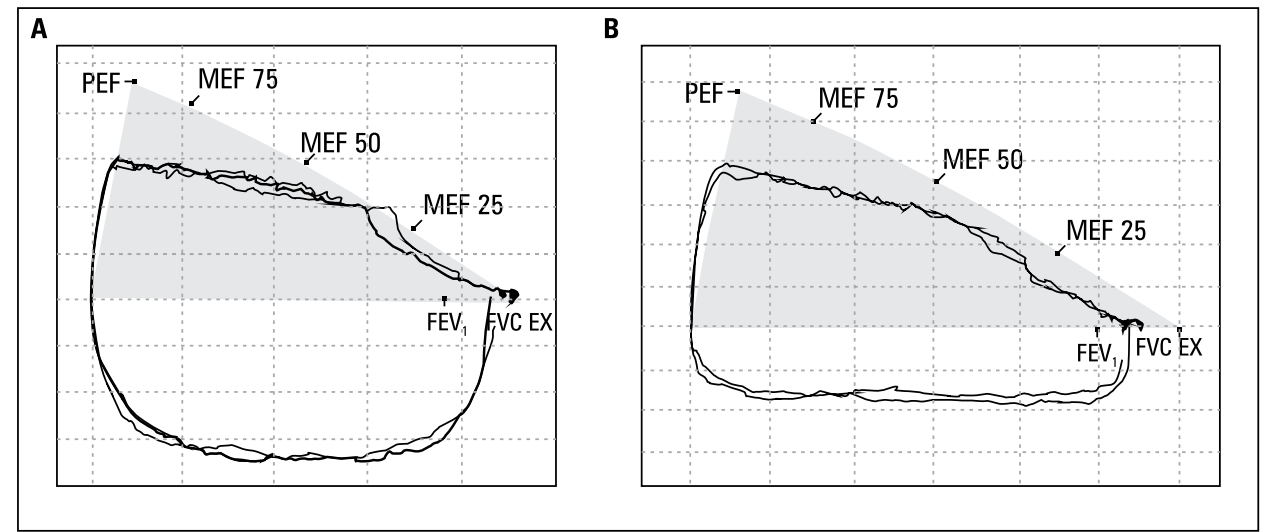

Figure 3. A - the flow-volume curve before and $15 \mathrm{~min}$ after inhalation of salbutamol) with a plateau of forced expiratory flow suggesting a central airway obstruction; B — the spirometry 17 months after the surgery

Table 2. Case 2 - values of spirometry parameters

\begin{tabular}{|c|c|c|c|c|}
\hline & \multicolumn{2}{|c|}{ Before surgery } & \multicolumn{2}{|c|}{17 months after surgery } \\
\hline & $\%$ of predicted value & $\begin{array}{l}\text { Standardised } \\
\text { Z-scores }\end{array}$ & $\%$ of predicted value & $\begin{array}{l}\text { Standardised } \\
\text { Z-scores }\end{array}$ \\
\hline FEV $_{1}$ & 97 & -0.28 & 93 & -0.67 \\
\hline FVC & 99 & -0.68 & 92 & -0.83 \\
\hline $\mathrm{FEV}_{1} \% \mathrm{FVC}$ & 90 & -1.20 & 96 & -0.52 \\
\hline
\end{tabular}

mitted to hospital. She was usually treated with inhaled bronchodilators and montelukast. She was also complaining about exercise intolerance due to dry cough on exertion.

On the admission day to our hospital, the girl was in a good condition, with normal height and weight, without dyspnea. Our diagnostic approach was initially asthma targeted - skin prick test was done (negative), spirometry was performed and showed abnormalities in the shape of flow-volume curve, which did not improve af- ter salbutamol. A plateau of the forced expiratory flow was presented suggesting a central airway obstruction (Fig. 3A, Table 2). Barium swallow $\mathrm{X}$-ray was done and revealed attenuation of the contrast on the posterior wall of the esophagus. Echocardiography and CT with intravenous contrast confirmed the diagnosis of vascular ring. The ring was built by right-sided aortic arch with the aberrant left subclavian artery.

The girl underwent surgery - vascular ring releasing with Kommerell's diverticulum plasty 
was carried out via posterolateral thoracotomy. Two months after the surgery the child was in a good condition, however, her spirometry did not improve yet (Fig. 3B, Table 2).

\section{Discussion}

Vascular rings can manifest in a wide range of symptoms - from severe respiratory distress to almost asymptomatic course. Moreover, scenario with the more serious symptoms allows to establish diagnosis in the younger age [1]. Diagnostic difficulties arise with patient age. Symptoms caused by vascular compression of the trachea and/or the esophagus can very easily mimic more common diseases like: asthma, gastro-esophageal reflux disease $[5,6]$ or even unspecified eating disorder [7].

Both our patients were as well misdiagnosed as having asthma. What changed the initial diagnosis, there were the results of spirometry despite that the values of spirometry parameters were within the normal range, the shape of the flow-volume curve was abnormal. Instead of characteristic concave shape of flow-volume curve for bronchial obstruction with reduced $\mathrm{FEV}_{1}$ and reduced $\mathrm{FEV}_{1} / \mathrm{FVC}$ ratio [8], there was a plateau of forced expiratory flow with normal $\mathrm{FEV}_{1}$ and decreased PEF. Such changes should alert to the possibility of central airway obstruction [9-11].

Typically, the pattern of a repeatable decreasing of forced expiratory flow, along without plateau of a forced inspiratory can be seen in a variable intrathoracic central airway obstruction. On the other hand, in a fixed central airway obstruction, the pattern of a repeatable plateau at a similar flow in both forced inspiratory and expiratory flows can be observed. Such conclusion cannot be drawn unless the patient makes the maximal efforts and the shapes of the maneuvers are repeatable [10].

Differential diagnosis of variable central airway obstruction should include the following: laryngeal polyp, large retrosternal goitre, while differential diagnosis of fixed central airway should include as follows: subglottic stenosis - congenital or acquired (for example after prolongated endotracheal intubation or in the course of autoimmune and inflammatory diseases like granulomatosis with polyangitis), vocal cord dysfunction, vocal cord paralysis, laryngeal web [9, 12].

The initial diagnosis of central airway obstruction should be subsequently followed by imaging methods. No gold standard for the diagnosis of vascular ring exists. Chest X-ray and barium swallow X-ray are used for the first step of the diagnostic workups. However, chest computed tomography, magnetic resonance imaging give more data about anatomy of vascular rings and its relation to adjacent structures [1, 13], whereas flexible bronchoscopy examination determines the degree of the tracheal compression and identifies tracheomalacia and/or bronchomalacia.

In some children, surgical correction of vascular rings can result not only in symptoms regression but also in improvement of pulmonary function. The obstruction presumably resolved with the child's growth. However, in some children (probably older ones) abnormalities of pulmonary function continue to stay, possibly due to persistent tracheomalacia [5].

In this report, we would like to emphasis the importance of spirometry for evaluating patients with asthma presumption. Especially in patients not responding to antiasthmatic treatment, careful examination of the shape of flow-volume loop can lead to the right diagnosis.

\section{Conflict of interest}

The authors declare no conflict of interest.

\section{References:}

1. Tola H, Ozturk E, Yildiz O, et al. Assessment of children with vascular ring. Pediatr Int. 2017; 59(2): 134-140, doi: 10.1111/ ped.13101, indexed in Pubmed: 27454661.

2. Bonnard A, Auber F, Fourcade L, et al. Vascular ring abnormalities: a retrospective study of 62 cases. J Pediatr Surg. 2003; 38(4): 539-543, doi: 10.1053/jpsu.2003.50117, indexed in Pubmed: 12677561.

3. Humphrey C, Duncan K, Fletcher S. Decade of experience with vascular rings at a single institution. Pediatrics. 2006; 117(5): e903-e908, doi: 10.1542/peds.2005-1674, indexed in Pubmed: 16585275.

4. McLaren CA, Elliott MJ, Roebuck DJ. Vascular compression of the airway in children. Paediatr Respir Rev. 2008; 9(2): 85-94, doi: 10.1016/j.prrv.2007.12.008, indexed in Pubmed: 18513668 .

5. Uchida DA. Late presentation of double aortic arch in school -age children presumed to have asthma: the benefits of spirometry and examination of the flow-volume curve. Respir Care. 2009; 54(10): 1402-1404, indexed in Pubmed: 19796421.

6. Calabrese C, Corcione N, Di Spirito V, et al. Recurrent respiratory infections caused by a double aortic arch: The diagnostic role of spirometry. Respir Med Case Rep. 2013; 8: 47-50, doi: 10.1016/j.rmcr.2012.12.006, indexed in Pubmed: 26029616.

7. Leipsic J, Correia DCS, Palmer R. A girl refuses to eat solid food because she is afraid of choking. Curr Psychiatr. 2016; 15(1): 35-44.

8. Moore VC. Spirometry: step by step. Breathe. 2012; 8(3): 232240, doi: 10.1183/20734735.0021711.

9. Hughes J. Pulmonary function: the basics. Medicine. 2008; 36(3): 142-150, doi: 10.1016/j.mpmed.2007.12.006.

10. Miller MR, Hankinson J, Brusasco V, et al. ATS/ERS Task Force. Standardisation of spirometry. Eur Respir J. 2005; 26(2): 319-338, doi: 10.1183/09031936.05.00034805, indexed in Pubmed: 16055882 . 
11. Pellegrino R, Viegi G, Brusasco V, et al. Interpretative strategies for lung function tests. Eur Respir J. 2005; 26(5): 948-968, doi: 10.1183/09031936.05.00035205, indexed in Pubmed: 16264058.

12. Mokoka MC, Ullah K, Curran DR, et al. Rare causes of persistent wheeze that mimic poorly controlled asthma. BMJ Case
Rep. 2013; 2013, doi: 10.1136/bcr-2013-201100, indexed in Pubmed: 24072840.

13. Gould SW, Rigsby CK, Donnelly LF, et al. Useful signs for the assessment of vascular rings on cross-sectional imaging. Pediatr Radiol. 2015; 45(13): 2004-16; quiz 2002, doi: 10.1007/ s00247-015-3424-7, indexed in Pubmed: 26260202. 